\title{
assessment of measurement equivalence with cross-national and longitudinal surveys in political science
}

gal ariely ${ }^{a}$ and eldad davidov ${ }^{b, *}$

${ }^{a}$ The Nanovic Institute for European Studies, University of Notre Dame, 46556 Notre Dame Indiana, USA

E-mail: galariely1976@gmail.com

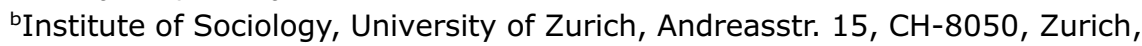
Switzerland

E-mail: davidov@soziologie.uzh.ch

${ }^{*}$ Corresponding author.

doi:10.1057/eps.2011.11; published online 15 April 2011

\section{Abstract}

This paper emphasises the importance of assessing measurement equivalence when using cross-national and longitudinal surveys. We illustrate how to test for measurement equivalence using multiple group confirmatory factor analysis and clarify under what conditions both meaningful comparisons of construct means and relationships between constructs are possible. Using data from the International Social Survey Programme, empirical applications dealing with attitudes towards welfare policies are provided to illustrate the procedure.

Keywords measurement equivalence; multiple group confirmatory factor analysis (MGCFA); cross-national and longitudinal surveys

\section{INTRODUCTION}

$\mathrm{P}$ roliferation of cross-national research projects in recent decades has enabled scholars to study public opinion under various political and societal contexts. Numerous political studies use data from cross-national surveys like the World Value Survey (WVS), the European
Social Survey (ESS), the International Social Survey Project (ISSP) and various other projects to study attitudes, beliefs or behaviour across contexts (Heath et al, 2005; Norris, 2009). Ironically, the very differences in contexts that give such cross-national studies their value also threaten the achievement of equivalence of concepts that are measured across 
different countries or time points. The ability to measure attitudes and beliefs in an equivalent way is, in principle, doubtful. To assume measurement equivalence of instruments designed to measure concepts across countries or over time may be misleading. Differences in regression coefficients or scale means across countries may derive from systematic biases of responses across countries or from variant understandings of the question items rather than from 'true' differences across groups (Horn and McArdle, 1992; Steenkamp and Baumgartner, 1998; Vandenberg and Lance, 2000; Van de Vijver, 2003a). Guaranteeing that the measurement of a relevant construct is equivalent across contexts consequently, constitutes a central concern when applying theories and instruments across different countries and/or over time (Billiet, 2003).

While this issue has received sizeable attention in disciplines like education, psychology, sociology and organisational behaviour, political scientists have often overlooked it. Awareness of the limitations of cross-national surveys is not new in political science. Nevertheless, there is insufficient concern about the issue in studies using cross-national or longitudinal survey data (Adcock and Collier, 2001; King et al, 2004).

The purpose of this paper is to address these problems and to provide a nontechnical introduction for establishing measurement equivalence ${ }^{1}$ using multiple group confirmatory factor analysis (MGCFA). Thus, we are not going to develop and present new methods to assess cross-national or longitudinal equivalence, nor give a technical presentation of the procedures, but rather review and present, in a non-technical way which is readable for the audience of this journal, existing methods to test for cross-national and longitudinal measurement equivalence. In the presentation, we focus on how to assess different degrees of measurement equivalence
The ability to measure attitudes and beliefs in an equivalent way is, in principle, doubtful. ...we focus on how to
assess different degrees
of measurement
equivalence according to
the purpose of the study.

according to the purpose of the study. For the illustration, we will use the Attitudes towards welfare policies scale that is part of the ISSP 'role of government' module. This module was conducted four times between 1985 and 2006 and across several countries, and its data was used in several studies (e.g., Anderson and Tverdova, 2003; Evans, 2007; Blekesaune and Quadagno, 2003; Lipsmeyer and Nordstrom, 2003; Papadakis and Bean, 1993). We decided to replicate the study of Svallfors $(2004,2006)$ and examined the cross-country equivalence in Britain, Germany (West), Sweden and the US, and, in addition, the longitudinal equivalence of the attitudes towards the welfare policies scale in Britain. ${ }^{2}$ By doing this, we will try to provide an answer to the question to what extent the scale is comparable across the four countries (space) and over time in Britain. For this purpose, we apply MGCFA. Before presenting our illustration, however, we shall first give a brief review of the problem of measurement equivalence in cross-national analyses and how this issue has been addressed in political science studies.

\section{CROSS-NATIONAL SURVEYS: POSSIBILITIES AND LIMITATIONS}

Scholars of politics used cross-national surveys as early as the 1950 s and the 
1960s, as illustrated by Almond and Verba's (1963) pioneering five-nation civic culture study. However, systematic cross-national survey projects were first launched in 1973 with the introduction of the Eurobarometer, and continued in the 1980s with projects like the European Values Survey, the WVS and the ISSP. Since the 1990s, we have witnessed the increasing proliferation of academic cross-national survey projects like the various regional barometers (e.g., ESS, Latinobarometer, Asiabarometer and Afrobarometer) and many other projects. Such projects led to the extension of survey research beyond Western countries to include areas such as Latin America and the post-communist states (for a review of cross-national survey projects see Heath et al, 2005; Kittilson, 2007; Norris, 2009).

These projects provide empirical tools for the systematic study of political values, attitudes and behaviour of people living in different political and social contexts. The data enable the assessment of the generalisability of theories and the examination of contextual influences on attitudes, behaviours and the relations between them. The expanding range of survey resources is, as Pippa Norris put it, 'One of the most dramatic recent developments, transforming the field of comparative politics during recent decades' (2009: 522). However, this line of social enquiry can also be considered a methodologically fragile endeavour.

Comparative inquiries that use crossnational surveys, but also experimental data or small-N samples, face the challenge of establishing equivalence, which is a requirement for meaningful comparisons. From the various types of equivalence that are relevant for this type of studies, we focus only on measurement equivalence (for a review of various equivalence types, see, e.g., Van Deth, 2009). Measurement equivalence is conceptually defined as 'whether or not, under different conditions of observing and studying phenomena, measurement operations yield measures of the same attribute' (Horn and McArdle, 1992: 117). In other words, the aim of establishing measurement equivalence is to assure that observed differences between groups are not misleading.

Political, linguistic and cultural differences that are fundamental for comparative analyses are a source of threat to the validity of measurements in any comparative analysis, as Van Deth aptly states: 'Establishing equivalence is one of the nastiest problems facing comparativists' (2009: 95). This is especially true (but not limited to) in the use of cross-national surveys. Naturally, using multiple languages in any project raises a question about the extent to which the meaning of a term is comparable to its original meaning after it has been translated to another language. Indeed, translation issues are one of the most frequently mentioned problems in establishing measurement equivalence in literature dealing with comparative surveys (e.g., Harkness et al, 2004; Davidov and De Beuckelaer, 2010). In addition, the comparability of cross-national surveys is challenged by further methodological problems like differences in survey practice that affect the sampling and coverage or differential scale use by the respondents (for a review see Van de Vijver, 2003a, b).

\section{CROSS-NATIONAL SURVEYS IN POLITICAL SCIENCE}

Many studies in political science employ cross-national surveys. Looking for the terms 'cross-national' and 'survey' at the JSTOR search engine in the political science category reveals over 1,800 papers. An examination of 82 crossnational studies that were published in 
nine major political science journals (the journals were: American Political Science Review, American Journal of Political Science, Comparative Political Studies, Public Opinion Quarterly, Political Studies, Political Analysis, British Journal of Political Science, Journal of Politics and the European Journal of Political Research) from 2000 to 2009 indicates that the issues that we have raised are rarely discussed in these studies. Less than half of the papers broached the issue of measurement and only about a quarter of the papers have tried to establish the equivalence of meaning across countries. Furthermore, studies that address the issue of measurement have often used the reliability measure of internal consistency (Cronbach's $\alpha$ ). This measure does not guarantee equivalence of meaning in different groups (but rather internal consistency). Furthermore, Cronbach's $\alpha$ is based on the assumption that all factor loadings and error variances are equal. Such assumptions have been sharply criticised as being unrealistic and, therefore, the use of Cronbach's $\alpha$ is neither recommended for testing scale reliability within a country nor for testing the comparability of a scale across countries or cultures (Raykov, 1997; Sijtsma, 2009).

Although several of these studies tried to assess the validity and reliability of the scales employed in their surveys, the claim that many studies still fail to pay sufficient attention to establishing measurement equivalence is relevant for many recent studies that utilised cross-national surveys. Applying ideas and techniques developed in the psychometrics literature regarding measurement equivalence is essential to bridge this gap. In the following sections, we will introduce the distinction between levels of measurement equivalence, clarify one of the most common ways to examine it and illustrate its use with examples.

\section{MEASUREMENT EQUIVALENCE}

In recent years, the increasingly greater consideration of item selection, translation and other procedures in cross-national surveys has enhanced the comparability of concepts across countries but this still cannot guarantee that we actually receive measures of the same attribute (e.g., Jowell et al, 2007, regarding the ESS). Guaranteeing equivalence requires statistical testing. Without such testing, the interpretation and comparison of results across groups is problematic (Billiet, 2003; Cheung and Rensvold, 2002; Hui and Triandis, 1985; for publications with a pragmatic/practical approach to the problems discussed above, see, e.g., Harkness et al, 2003; Harkness et al, 2010; or Van Deth, 2009).

Various techniques have been developed to test for equivalence, including item response theory, latent class analysis and multidimensional scaling (e.g., Davidov et al, 2011b; De Beuckelaer, 2005; Van de Vijver, 2003b). The most common approach to testing cross-national or longitudinal measurement equivalence is probably the MGCFA model (Jöreskog, 1971; Bollen, 1989; Steenkamp and Baumgartner, 1998). Several studies have used MGCFA to assess measurement equivalence of scales that are part of the ESS and ISSP, like interpersonal, social and political trust (Reeskens and Hooghe, 2008; Van der Veld and Saris, 2011), human values (Davidov et al, 2008; Davidov, 2008, 2010a), ethnocentrism and authoritarianism (Billiet, 2003), attitudes towards immigration (Meuleman et al, 2009), public support for democracy (Ariely and Davidov, 2010) as well as nationalism and constructive patriotism (Davidov, 2009, 2010b).

\section{LEVELS OF EQUIVALENCE}

Steenkamp and Baumgartner (1998) and Vandenberg and Lance (2000) offer 
procedural guidelines to facilitate the assessment of measurement equivalence in cross-national or longitudinal studies using MGCFA. They propose at least three important hierarchical levels of measurement equivalence: configural, metric and scalar. The distinction between these three levels enables them to match the assessed level of equivalence with the research purpose.

Configural equivalence constitutes the lowest level of equivalence. It requires that the items in the measuring instrument exhibit the same configuration of factor loadings (between the latent variable and the items) in each group (country, culture or time point). In other words, the confirmatory factor analysis (CFA) has to assess whether the same items measure each latent variable in all the groups covered in the study. Configural equivalence is supported if: (a) a model specifying the items measuring each latent variable fits the data well; (b) all factor loadings are substantial and significant; and (c) no large modification indices exist that point to model misspecifications.

Metric equivalence is a higher level of equivalence that provides an indication that people in different countries or time points understand the questions similarly. It requires that the factor loadings between items and constructs are equal. It is tested by restricting the factor loading of each indicator on its corresponding construct to be equal across groups. Metric equivalence is required to compare constructs' unstandardised correlates (covariances, unstandardised regression coefficients) with other variables of interest meaningfully across groups. It is supported if the model cannot be significantly improved by removing any of the constraints. To enable cross-national or longitudinal comparisons, however, it is not necessary that all factor loadings be equal across groups. Several scholars (Byrne et al, 1989; Steenkamp and
Baumgartner, 1998) have suggested that two equal factor loadings per construct are sufficient to allow a comparison of effects. This is known as partial metric equivalence.

Scalar equivalence represents an even higher level of equivalence. While factor loadings are kept equal across groups in order to establish metric equivalence, the scalar equivalence test is even stricter, requiring, in addition, equal indicator intercepts across groups (Meredith, 1993). Scalar equivalence guarantees that cross-national or longitudinal differences in the means of the observed items are the consequence of differences in the means of their corresponding constructs and not due to differences in factor loadings or indicator intercepts (Steenkamp and Baumgartner, 1998). Thus, findings of scalar equivalence may allow meaningful mean comparisons of constructs. In assessing scalar equivalence, the intercepts of the underlying indicators are required to be equal across groups. Scalar equivalence is supported if the model fits the data well and cannot be improved by removing any of the equality constraints. Also in this respect, several scholars (Byrne et al, 1989; Steenkamp and Baumgartner, 1998) have suggested that two equal intercepts per construct are sufficient to allow a comparison of means. In this case, the indicators of these intercepts must have, in addition, equal factor loadings. They termed this situation partial scalar equivalence.

The distinction between these three levels enables the researcher to decide which level of equivalence is required given the goals of the cross-national study. If the aim of the study is to examine whether a construct can be conceptualised in the same way across countries, configural equivalence is sufficient. If the study is designed to examine the relationships between different constructs, that is, to conduct multivariate analyses, and compare these 
relationships across countries or time points, metric equivalence is required. When the research aim is to make a comparison of means, scalar equivalence is needed (Steenkamp and Baumgartner, 1998).

\section{EMPIRICAL ILLUSTRATION}

We now present examples to illustrate the way to assess measurement equivalence in a cross-national or longitudinal survey using MGCFA. Our illustrations deal with attitudes towards welfare policies.

\section{DATA}

We have chosen to use data from the ISSP module 'role of government'. This module has been used in several studies that explore cross-national variations in attitudes towards the role of government. Furthermore, this module has been conducted four times (1985, 1990, 1996, 2006) (for further details and documentation of the ISSP module, see http://www.issp.org/data.shtml and http://zacat.gesis.org/webview/index.jsp). Using this module enables us to illustrate equivalence assessment also across time points and not only across countries. In order to simplify the illustration, from the various studies that have used the scale, we have chosen to replicate Svallfors' (2004; see also 2006) studies that compare four countries from the 1996 survey: The US $(N=1,332)$, Britain $(N=989)$, Germany (West) $(N=2,361)$ and Sweden $(N=1,238)$. Theoretically, Svallfors analysed these four countries since they exhibit differences in the design of their welfare regimes. We have chosen to analyse the scale's equivalence across four time points from 1985 to 2006 in Britain. Findings of measurement equivalence will enable the analysis of changes in Britain across time in attitudes towards welfare policies which is relevant, as this country's welfare regime shifted dramatically in the 1980s under Margaret Thatcher. The way questions are understood may also change within a culture, and this is more likely to happen with an everincreasing time gap between the surveys. Therefore, before relations to other concepts and means are analysed and compared over time, it is necessary to also test whether reasonable longitudinal equivalence of the concept holds.

\section{MEASURES}

Following Svallfors (2004), we used eight items to measure attitudes towards welfare policies. Each item on this scale is rated on a 4-point Likert scale (with lower numbers indicating stronger support). ${ }^{3}$ The measurement model is portrayed in Figure 1.

\section{SINGLE-COUNTRY ANALYSES}

In line with Byrne's (2010) assertion regarding the importance of conducting single-group analyses prior to multiplegroup comparisons, we began with four separate CFAs for each country on the 1996 data. First, we computed four Pearson (product moment) covariance matrices (unstandardised), one for each of the four countries, as input for estimating the CFAs. Pairwise deletion was used to deal with missing values in the data (the average rate of missing data was rather moderate: 4.9 per cent in Britain, 6.2 per cent in Germany (West), 6.3 per cent in Sweden and 8.5 per cent in the US sample. For the CFA analysis, we employed the Amos 16.0 software package (Arbuckle, 2007) and the maximumlikelihood procedure (for further guidance on how to use Amos, see Arbuckle, 2007; Byrne, 2010, and the software websites at http://amosdevelopment.com/index .htm and http://spss.com/amos/). In a final step, we repeated the analysis using the full information maximum likelihood 


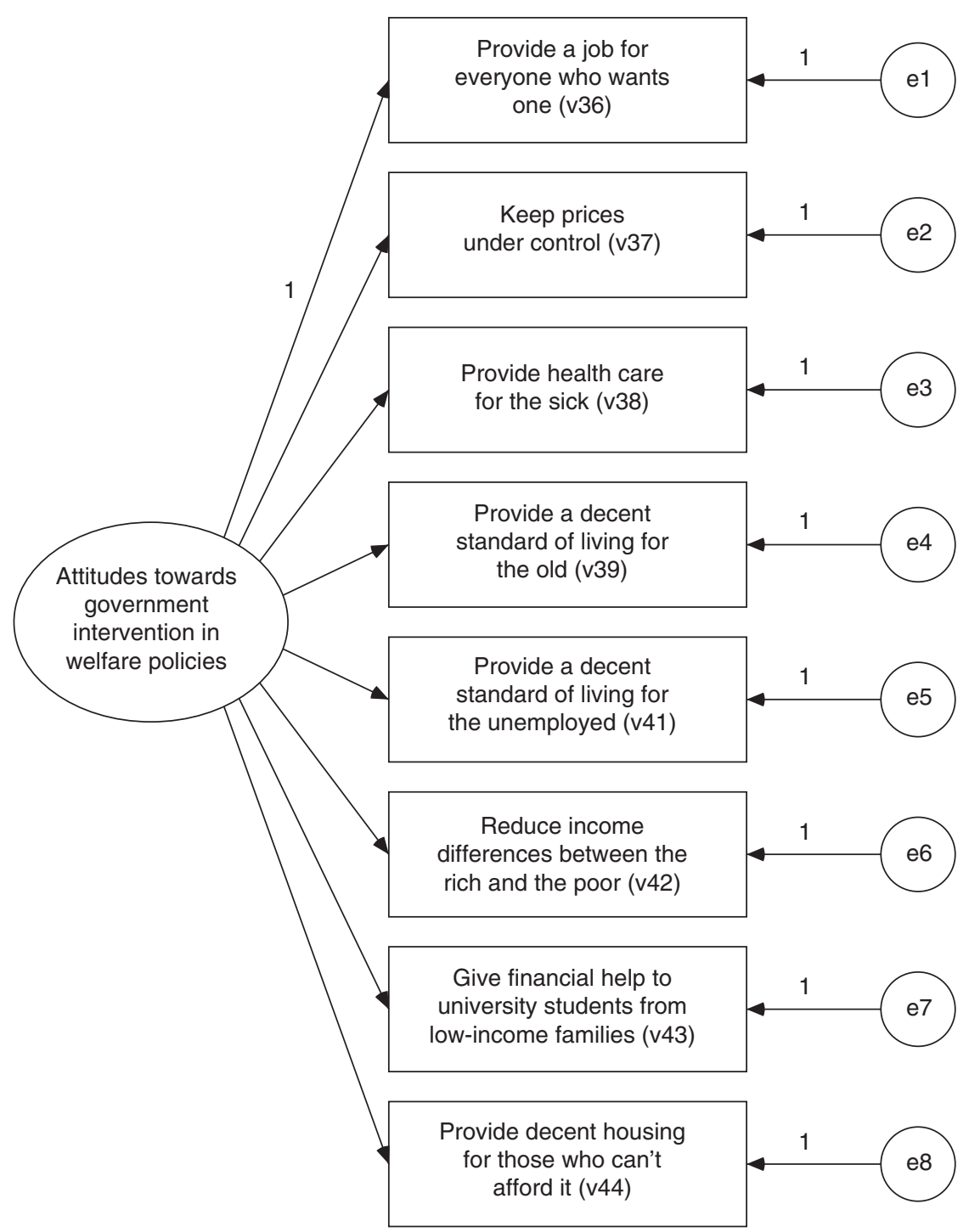

Figure 1 Attitudes towards government intervention in welfare policies.

procedure which is recommended by various authors to deal with the problem of missing values (see, e.g., Schafer and Graham, 2002) to ascertain whether our conclusions remain stable. The fit of the model in each of the single-country analyses was assessed using three fit indices: the comparative fit index - CFI, the root mean square error of approximation RMSEA and the probability of close fit Pclose. We adopted these fit indices in order to discern between well-fitting and poorly fitting models (for a comprehensive review of model fit indices criteria, see Brown, 2006). When the RMSEA value is smaller than 0.05 and the Pclose value is larger than 0.5 , the model can be assumed to demonstrate a good fit to the data. CFI values larger than 0.95 provide further indication of a good model fit (Hu and Bentler, 1999; Marsh et al, 2004). Since the sample size is large and 
the $p$ value may consequently reject models with only small misspecifications, we were unable to rely on the $p$ value in selecting a model.

The single-country analyses reveal modest differences in the measurement model across countries. Modification indices in the output reveal that some error correlations should be released. This indicates that some aspects of welfare policy are perceived to be more strongly linked to each other in some countries compared with others. Some of these required modifications are different across countries. For example, whereas in the US there is a strong correspondence between providing a decent standard of living for the old and for the unemployed in the public's eyes, there is no such strong link evident in the Swedish sample. Providing a decent standard of living for the unemployed is more strongly related to providing decent housing for those who cannot afford it, in the eyes of the German public compared with other countries. Allowing for different error correlations in different countries may result in different model configurations across countries and even imply the absence of full configural invariance. Furthermore, allowing so many items to load on a single concept risks mixing up several dimensions, whereas some researchers may be interested in treating these dimensions separately. Thus, we decided to modify the eight-item scale used by Svallfors $(2004,2006)$ by dropping items that require the aforementioned modifications. This procedure led to a new scale that used only four items: 'Providing a job for everyone who wants one' (V36); 'Providing health care for the sick' (V38); 'Providing a decent standard of living for the old' (V39); and 'Providing a decent standard of living for the unemployed' (V41). From a theoretical point of view, changing the items in the scale also changes the meaning of the measured construct. The new modified scale has a more narrow meaning that refers to helping the needy (sick, elderly, unemployed), while the previous scale was broader and measured also issues like reducing income differences between the rich and the poor. These are different aspects of welfare policy. The new scale, however, remains rather similar to the original one. The advantage of the new scale is that it taps a somewhat narrower content, its items function more similarly across countries and its configuration is more compatible for comparative analysis.

In all countries, the modified scale requires freeing a correlation between the errors of the items 'Providing health care for the sick' (e3) and 'Providing a decent standard of living for the old' (e4). In other words, the two aspects are closely related in all countries. Table 1 reports the global fit measures in each country. This model displays an acceptable or a good fit in each country.

An examination of the factor loadings of the items reveals that the items display substantial factor loadings on their corresponding concept (Brown, 2006), and all of them are highly significant in all countries (a summary of the factor loadings for the single country analyses may be obtained from the first author upon request).

\section{MULTIPLE-GROUP CFAS AND TESTING FOR EQUIVALENCE}

To test whether equivalence across countries holds, we will employ a bottom-up procedure (Vandenberg and Lance, 2000). Specifically, we begin with the least constrained MGCFA model (configural equivalence). Then we gradually increase the number of constraints and assess whether the model fit deteriorates significantly when assessing metric and scalar equivalence. This process stops when the model fit is not acceptable (a top-down approach would imply 
Table 1: Single-country analyses: Modifications and global fit for attitudes towards government intervention scale

\begin{tabular}{lccccc}
\hline Country & $\chi^{2}$ & $\begin{array}{c}\text { Degrees of } \\
\text { freedom }\end{array}$ & CFI & RMSEA & Pclose \\
\hline US & 6.1 & 1 & 0.996 & 0.065 & 0.23 \\
Britain & 0.0 & 1 & 1.000 & 0.000 & 0.95 \\
Germany (West) & 3.3 & 1 & 0.999 & 0.032 & 0.71 \\
Sweden & 0.0 & 1 & 1.000 & 0.000 & 0.99 \\
\hline
\end{tabular}

Note: Data is taken from the ISSP 1996. $\chi^{2}$ values are rounded up. CFI - comparative fit index; RMSEA - root mean square error of approximation; Pclose - probability of close fit.

Table 2: Fit measures of the MGCFA (attitudes towards government intervention) across the US, Britain, Germany (West) and Sweden

\begin{tabular}{lrcccr}
\hline Model & $\chi^{2}$ & $\begin{array}{c}\text { Degrees of } \\
\text { freedom }\end{array}$ & CFI & RMSEA & Pclose \\
\hline 1. Configural equivalence & 9.4 & 4 & 0.999 & 0.016 & 1.00 \\
2. Full metric equivalence & 116.4 & 13 & 0.979 & 0.038 & 0.99 \\
3. Partial metric equivalence & 23.5 & 7 & 0.997 & 0.021 & 1.00 \\
4. Partial scalar equivalence & 58.7 & 10 & 0.990 & 0.030 & 1.00 \\
\hline
\end{tabular}

Note: Data is taken from the ISSP 1996. $\chi^{2}$ values are rounded up. For abbreviations of global fit measures, see Table 1.

${ }^{a}$ The constraints of items V36 and V41 were released.

exactly the opposite procedure). For model comparison, we applied the 'practical perspective' (Byrne and Stewart, 2006: 307). Based on Cheung and Rensvold (2002) and Chen (2007), the decrease in CFI should not exceed 0.01 when additional constraints are included in the model. ${ }^{4}$

The results of the equivalence tests are summarised in Table 2. Based on the results for the configural equivalence model (Model 1 ) reported in the first row, we cannot reject this model. In other words, the construct configuration can be considered as invariant across Britain, Germany, Sweden and the US. The second row in Table 2 reports the fit indices of the full metric equivalence model (Model 2), which requires equality between the factor loadings of the scale indicators across the four countries. It turns out that the decrease in CFI (0.02) is above the cut-off criteria (Chen, 2007), and thus we reject the full metric invariance model. To assess partial metric invariance, we released the cross-country equality constraints of the factor loadings for items V36 (providing a job for everyone who wants one) and V41 (providing a decent standard of living for the unemployed) on the grounds that the factor loadings of these indicators displayed the most severe violations of equivalence. Thus, we have created a partial metric equivalence model (Model 3). Compared with the configural equivalence model, the CFI difference reduced to 0.002 and, thus, this model is supported by the data. Differences in the size of the factor loading of V36 and V41 may 
indicate the different role that these items play in attitudes towards welfare policies in the different countries.

Next, we tested a partial scalar equivalence model (Model 4), where intercepts for the items V38 and V39 were constrained to be equal across countries (the intercepts of V36 and V41 were free, since the factor loadings for these items were also released). The decrease of CFI $(\Delta \mathrm{CFI}=0.007)$ compared with the partial metric equivalence model is acceptable (Chen, 2007). Hence, the partial scalar model is acceptable as well. In this partial scalar equivalence model, two items (V38 and V39) have equal factor loadings and intercepts across countries.

In summary, the findings indicate that partial scalar equivalence holds for the four countries. In other words, not only is the meaning of the constructs as measured by the chosen indicators probably similar in these countries, but one can also compare mean scales between countries meaningfully. To compare the means, one should compare the means of the latent variables in the partial scalar equivalence model (Model 4). It turns out that the latent means for Britain, Germany, Sweden and the US are 1.2, $1.5,1.3$ and 1.8 , respectively (rated on a scale from 1 to 4 with lower numbers indicating stronger support). Thus, in Britain, respondents are most supportive of government intervention in welfare policies, which may be accounted for by major shifts in the welfare system in the 1980s that met strong opposition among the population. In the US, people are least supportive and in Germany and Sweden the attitude levels are in-between. Thus, establishing full or partial scalar equivalence provides us with the legitimacy to conduct a mean comparison meaningfully.

\section{EQUIVALENCE ACROSS TIME}

As we have noted, one of the advantages of cross-national survey projects is their

\section{Establishing partial scalar equivalence ... provides us with the legitimacy to conduct such a longitudinal mean comparison meaningfully.}

repetition across the years, enabling scholars to trace longitudinal changes in public attitudes on the macro level. While most of the dangers to equivalence (e.g., language, cultural differences) do not exist in a study that is conducted in the same country, assessment of equivalence before studying changes in scale means across time is nevertheless required, especially if the study examines changes over a relatively long period of time. After all, the meaning of concepts and the way their scale is used may change over the years. In this assessment, each survey year is one group in the MGCFA model.

Let us look, for example, at the longitudinal equivalence of the government intervention scale in Britain in the survey years 1985, 1990, 1996 and 2006. According to the procedure illustrated above, we conducted a MGCFA and tested for equivalence between those four time points to examine whether this scale can be used for a meaningful longitudinal comparison of means. Indeed, it turned out that partial scalar invariance can be established. Thus, change over time may be meaningfully estimated in Britain using the partial scalar invariance model. Findings suggest that support for government intervention in welfare policies in Britain displayed a consistent, albeit slight decrease over time. Establishing partial scalar equivalence in this case also provides us with the legitimacy to conduct such a longitudinal mean comparison meaningfully. ${ }^{5}$ 


\section{CONCLUSION AND IMPLICATIONS}

Cross-national surveys provide researchers with important information for the study of politics, yet these are influenced by the inherent methodological challenges of comparability. Although Adcock and Collier (2001: 534) argue that the potential difficulty that context poses for valid measurement and the related task of establishing measurement equivalence across diverse units deserve more attention in political science', our review of cross-national studies published between 2001 and 2009 in leading journals revealed that their call has not been addressed satisfactorily. As cross-national survey data are becoming more and more prominent in the study of politics, this issue needs further attention. Recent cross-national survey projects have put great effort into increasing cross-national measurement equivalence by applying high standards of data collection, improving translation procedures and striving for higher response rates. However, the application of such high standards in the data collection procedures is not enough to guarantee that measurement scales are invariant across cultures. In this paper, we have stressed the importance of statistically establishing measurement equivalence to comparative analysis and have demonstrated how measurement equivalence can be examined in cross-national and/or longitudinal data. Establishing measurement equivalence is not a goal in itself. Nonetheless, lack of measurement equivalence is a source of bias and endangers the meaningfulness of cross-country or longitudinal comparisons.

Our illustration showed how neglecting the issue of measurement equivalence of a scale may lead one to use a scale that is not configurally invariant across countries. This is not only a technical issue, because one runs into the risk that

\section{... lack of measurement equivalence is a source of bias and endangers the meaningfulness of cross-country or longitudinal comparisons.}

\author{
MGCFA analysis \\ is an efficient way to \\ examine measurement \\ equivalence.
}

comparisons of such a scale may not be meaningful. Our analysis has shown that the slightly modified scale measuring attitudes towards welfare policies may be compared across Britain, Germany, Sweden and the US. Researchers can now try to explain these attitudes using socio-demographic or other explanatory variables (e.g., Svallfors, 2004: 126), use these attitudes to explain behaviour (e.g., voting) and compare these effects across countries with confidence. Furthermore, the means of these attitudes towards welfare policies may also be meaningfully compared, and differences in the means could be attributed to different histories, economic or social policies. The illustration shows how the means of the latent variable may be compared rather than the single indicators. Such analyses could not be conducted with confidence without prior tests of equivalence.

The paper has shown that conducting MGCFA analyses is an efficient way to examine measurement equivalence, and this procedure can be applied by the researcher even during the process of the selection of items, which measure his/her theoretical concepts. This method will help to determine which indicators load on the theoretical construct of interest across all countries or time units. 
As we have pointed out, the level of equivalence that is required is determined by the study goals. It is important to note, however, that full or partial scalar equivalence are not commonly achieved.

When no type of measurement equivalence is present in the data, there are certain strategies one could consider:

a. If no configural equivalence is present, one may select those items that load significantly on the theoretical construct of interest across all countries or time points and drop items that do not function well. While dropping items, one should be aware of the fact that the content of the measured theoretical construct might change due to the elimination of some of its measurements.

b. Furthermore, lack of invariance for a certain item might deliver important information about ways in which certain societies differ in the understanding of specific concepts. For example, the different factor loadings and intercepts of the items V36 and V41 in our study (providing a job and a decent standard of living for the unemployed) instructs us that these two aspects are perceived quite differently across countries; providing a job is far more important in Germany and the US compared with the other countries, and providing a decent standard of living for the unemployed is considerably less important in Britain compared with the other countries as indicated by the lower factor loading. Thus, lack of invariance can deliver important information as to how individuals in various societies differ in the way they understand certain questions.

c. If no metric or scalar equivalence are evident, one may fall back on partial metric or scalar equivalence as in the present study. Partial equivalence will allow meaningful cross-country comparisons. d. When even partial metric or scalar equivalence is not present, one might consider dropping the deviating countries and conducting a cross-national analysis across a smaller set of countries where equivalence holds. Such a selection of countries allows a meaningful comparison that would not be possible when including all the countries in the analysis.

One may ask whether all the work is worth the effort, especially when some forms of metric and scalar invariance are eventually established. The methodological literature suggests that we do not know in advance, whether our endeavours of comparative analysis are appropriate, unless we commit ourselves to the effort of conducting measurement equivalence tests. Only then do we know if the scales that we use are comparable or not, and if findings in our comparative study are indeed meaningful. When no measurement equivalence test is conducted, we remain uncertain about whether our comparisons are valid. Additional tests of construct validity should accompany any tests of measurement equivalence to guarantee the validity of our findings in each country.

In an ideal situation, we would need three to four good items that capture the content of the theoretical constructs of interest well. However, in practice, this ideal is often not met. Cross-national survey projects are planned to cover various areas of interest, and there are limits to the number of questions that can be combined in one questionnaire. It is quite common in cross-national surveys to measure complex constructs with only a few items or even with single item measures. Therefore, if a scholar wants to drop items that are not invariant across countries or even over time, s/he might lack sufficient items for this procedure. Hopefully, the continuing attempts to improve cross-national surveys will 
broaden the use of multi-item scales. This in turn will increase the potential of MGCFA analyses or other methods for testing equivalence. Until then, we believe that researchers using crossnational and/or longitudinal survey data (or other sorts of comparative data) should implement available techniques to test for equivalence when several items are used to measure their theoretical constructs of interest.

\section{Acknowledgements}

\author{
We would like to thank Lisa Trierweiler \\ for the English proof of the manuscript.
}

\section{Notes}

1 The terms equivalence and invariance are used in the literature interchangeably. In this study, we consistently use the term equivalence. Throughout the text, we use the term 'cross-national' consistent with our empirical illustration. However, the method we illustrate applies for any comparison across groups (such as males and females, age or cultural groups).

2 In this study, we focus on tests of cross-country equivalence and comparability. However, it should be noted that construct validity should be tested prior to any cross-country scale comparisons. The reader is referred to Svallfors (2004).

3 Lubke and Muthén (2004) argue that an analysis of Likert data under the assumption of multivariate normality may be problematic. However, several studies have shown using simulations or real data that using Likert scales and skewed data does not necessarily affect the probability of incorrect conclusions in MGCFA and that other strategies have different problems (see, e.g., Davidov et al, 2011a; De Beuckelaer, 2005; Welkenhuysen-Gybels and Billiet, 2002; Welkenhuysen-Gybels, 2004). Other software packages, such as Lisrel (Jöreskog and Sörbom, 2001) or Mplus (Muthén and Muthén, 2007) offer alternative estimation procedures to deal with Likert and ordinal scales, but they are more difficult to use than Amos. 4 Saris et al (2009) propose a different approach to evaluate model fit, which is based on the modification indices, the statistical power and the expected parameter change.

5 Obviously, a similar analysis could be conducted to replicate Svallfors (2004: 127-30) and its longitudinal analysis in Sweden.

\section{References}

Adcock, R. and Collier, D. (2001) 'Measurement validity: A shared standard for qualitative and quantitative research', American Political Science Review 95(3): 529-546.

Almond, G.A. and Verba, S. (1963) The Civic Culture: Political Attitudes and Democracy in Five Nations, Princeton: Princeton University Press.

Anderson, C.J. and Tverdova, Y.V. (2003) 'Corruption, political allegiances, and attitudes toward government in contemporary democracies', American Journal of Political Science 47: 91-109.

Arbuckle, J.L. (2007) Amos 16.0 User's Guide, Chicago: SPSS.

Ariely, G. and Davidov, E. (2010) 'Can we rate public support for democracy in a comparable way? Crossnational equivalence of democratic attitudes in the world value survey', Social Indicators Research, doi: $10.1007 /$ s11205-010-9693-5.

Billiet, J. (2003) 'Cross-Cultural Equivalence with Structural Equation Modeling', in J.A. Harkness, F.J.R. Van de Vijver and P.H. Mohler (eds.) Cross-Cultural Survey Methods, New York: John Wiley \& Sons, pp. 247-264.

Blekesaune, M. and Quadagno, J. (2003) 'Public attitudes toward welfare state policies. A comparative analysis of 24 nations', European Sociological Review 19(5): 415-427.

Bollen, K.A. (1989) Structural Equations with Latent Variables, New York: Wiley.

Brown, T.A. (2006) Confirmatory Factor Analysis for Applied Research: Methodology in the Social Sciences, New York: Guilford Press.

Byrne, B.M. (2010) Structural Equation Modeling with AMOS: Basic Concepts, Applications, and Programming, 2nd edn., New York: Routledge.

Byrne, B.M., Shavelson, R.J. and Muthén, B. (1989) 'Testing for the equivalence of factor covariance and mean structures: The issue of partial measurement invariance', Psychological Bulletin 105(3): 456-466.

Byrne, B.M. and Stewart, S.M. (2006) 'The MACS approach to testing for multigroup invariance of a second-order structure: A walk through the process', Structural Equation Modeling 13(2): 287-321. 
Chen, F.F. (2007) 'Sensitivity of goodness of fit indexes to lack of measurement invariance', Structural Equation Modeling 14(3): 464-504.

Cheung, G.W. and Rensvold, R.B. (2002) 'Evaluating goodness-of-fit indexes for testing measurement invariance', Structural Equation Modeling 9(2): 233-255.

Davidov, E. (2008) 'A cross-country and cross-time comparison of the human values measurements with the second round of the European social survey', Survey Research Methods 2(1): 33-46.

Davidov, E. (2009) 'Measurement equivalence of nationalism and constructive patriotism in the ISSP: 34 countries in a comparative perspective', Political Analysis 17(1): 64-82.

Davidov, E. (2010a) 'Testing for comparability of human values across countries and time with the third round of the European social survey', International Journal of Comparative Sociology 51(3): 171-191.

Davidov, E. (2010b) 'Nationalism and patriotism: A longitudinal analysis in 22 countries with the ISSP', International Journal of Public Opinion Research, 22(4): 485-510.

Davidov, E., Datler, G., Schmidt, P. and Schwartz, S.H. (2011a) 'Testing the Invariance of Values in the Benelux Countries with the European Social Survey: Accounting for Ordinality', in E. Davidov, P. Schmidt and J. Billiet (eds.) Cross-Cultural Analysis: Methods and Applications, New Jersey: Routledge.

Davidov, E. and De Beuckelaer, A. (2010) 'Testing the equivalence of an instrument to assess Schwartz' human values: How harmful are translations?' International Journal of Public Opinion Research, doi: $10.1093 /$ ijpor/edq030.

Davidov, E., Schmidt, P. and Billiet, J. (2011b) Cross-Cultural Analysis: Methods and Applications, New Jersey: Routledge.

Davidov, E., Schmidt, P. and Schwartz, S.H. (2008) 'Bringing values back in: The adequacy of the European social survey to measure values in 20 countries', Public Opinion Quarterly 72(3): 420-445.

De Beuckelaer, A. (2005) 'Measurement invariance issues in international management research', Ph.D. dissertation, Limburgs Universitie Centrum, Limburg, the Netherlands.

Evans, G. (2007) 'Britain and Europe: Separate worlds of welfare?' Government and Opposition 33(2): $183-198$.

Harkness, J.A., Braun, M., Edwards, B., Johnson, T.P., Lyberg, L., Mohler, P.Ph., Pennell, B.-E. and Smith, T.W. (2010) Survey Methods in Multicultural, Multinational, and Multiregional Contexts, New York: Wiley.

Harkness, J., Pennell, B.-E. and Schoua-Glusberg, A. (2004) 'Survey Questionnaire Translation and Assessment', in S. Presser et al (eds.) Methods for Testing and Evaluating Survey Questionnaires, New York: Wiley, pp. 453-473.

Harkness, J.A., Van de Vijver, F.J.R. and Mohler, P.P. (2003) Cross-Cultural Survey Methodology, New York: Wiley.

Heath, A., Fisher, S. and Smith, S. (2005) 'The globalization of public opinion research', Annual Review of Political Science 8: 297-333.

Horn, J.L. and McArdle, J.J. (1992) 'A practical and theoretical guide to measurement invariance in aging research', Experimental Aging Research 18(3): 117-144.

Hu, L. and Bentler, P.M. (1999) 'Cutoff criteria for fit indexes in covariance structure analysis: Conventional criteria versus new alternatives', Structural Equation Modeling 6(4): 1-55.

Hui, C.H. and Triandis, H.C. (1985) 'Measurement in cross-cultural psychology: A review and comparison of strategies', Journal of Cross-Cultural Psychology 16(2): 131-152.

Jöreskog, K.G. (1971) 'Simultaneous factor analysis in several populations', Psychometrika 36(4): 409-426.

Jöreskog, K.G. and Sörbom, D. (2001) Lisrel 8: User's Reference Guide, Chicago: Scientific Software International.

Jowell, R., Roberts, C., Fitzgerald, R. and Gillian, E. (eds.) (2007) Measuring Attitudes Cross-Nationally: Lessons from the European Social Survey, London: Sage Publications.

King, G., Murray, C.J.L., Salomon, J.A. and Tandon, A. (2004) 'Enhancing the validity and cross-cultural comparability of measurement in survey research', American Political Science Review 97(4): 567-583.

Kittilson, M.C. (2007) 'Research Resources in Comparative Political Behavior', in J.R. Dalton and H. Klingemann (eds.) Oxford Handbook of Political Behavior, Oxford: Oxford University Press, pp. 865-895.

Lipsmeyer, C. and Nordstrom, T. (2003) 'East versus west: Comparing political attitudes and welfare preferences across European societies', Journal of European Public Policy 10(3): 339-364.

Lubke, G.H. and Muthén, B.O. (2004) 'Applying multigroup confirmatory factor models for continuous outcomes to Likert scale data complicates meaningful group comparisons', Structural Equation Modeling 11(4): 514-534. 
Marsh, H., Hau, K.T. and Wen, Z. (2004) 'In search of golden rules: Comment on hypothesis-testing approaches to setting cutoff values for fit indexes and dangers in overgeneralizing Hu and Bentler's (1999) findings', Structural Equation Modeling 11(3): 320-341.

Meredith, W. (1993) 'Measurement invariance, factor analysis and factorial invariance', Psychometrika 58(4): 525-543.

Meuleman, B., Davidov, E. and Billiet, J. (2009) 'Changing attitudes toward immigration in Europe, 2002-2007. A dynamic group conflict theory approach', Social Science Research 38(2): 352-365.

Muthén, L.K. and Muthén, B.O. (2007) Mplus User's Guide, Los Angeles, CA: Muthén \& Muthén.

Norris, P. (2009) 'The Globalization of Comparative Public Opinion Research', in T. Landman and N. Robinson (eds.) The Sage Handbook of Comparative Politics, London: Sage, pp. 522-540.

Papadakis, E. and Bean, B. (1993) 'Popular support for the welfare state: A comparison between institutional regimes', Journal of Public Policy 13(3): 227-254.

Raykov, T. (1997) 'Scale reliability Cronbach's coefficient alpha, and violations of essential tauequivalence with fixed congeneric components', Multivariate Behavioral Research 32(4): 329-353.

Reeskens, T. and Hooghe, M. (2008) 'Cross-cultural measurement equivalence of generalized trust. Evidence from the European social survey (2002 and 2004)', Social Indicators Research 85(3): 515-532.

Saris, W.E., Satorra, A. and Van der Veld, W. (2009) 'Testing structural equation models or detection of misspecifications?' Structural Equation Modeling 16: 561-582.

Schafer, J.L. and Graham, J.W. (2002) 'Missing data: Our view of the state of the art', Psychological Methods 7(2): 147-177.

Sijtsma, K. (2009) 'On the use, the misuse, and the very limited usefulness of Cronbach's alpha', Psychometrika 74(1): 107-120.

Steenkamp, J.-B.E.M. and Baumgartner, H. (1998) 'Assessing measurement invariance in cross-national consumer research', Journal of Consumer Research 25(1): 78-107.

Svallfors, S. (2004) 'Class, attitudes and the welfare state: Sweden in comparative perspective', Social Policy and Administration 38(2): 119-138.

Svallfors, S. (2006) The Moral Economy of Class: Class and Attitudes in Comparative Perspective, Stanford, CA: Stanford University Press.

Van Deth, J.W. (2009) 'Establishing Equivalence', in T. Landman and N. Robinson (eds.) The SAGE Handbook of Comparative Politics, London: Sage Publications, pp. 84-100.

Van de Vijver, F.J.R. (2003a) 'Bias and Equivalence: Cross-Cultural Perspective', in J.A. Harkness, F.J.R. Van de Vijver and P.H. Mohler (eds.) Cross-Cultural Survey Methods, New York: John Wiley \& Sons, pp. 143-155.

Van de Vijver, F.J.R. (2003b) 'Bias and Substantive Analyses', in J.A. Harkness, F.J.R. Van de Vijver and P.H. Mohler (eds.) Cross-Cultural Survey Methods, New York: John Wiley \& Sons, pp. 207-233.

Van der Veld, W. and Saris, W.E. (2011) 'Methodological Aspects of the Cross-National Evaluation of a Theory on the Causes of Generalized Social Trust', in E. Davidov, P. Schmidt and J. Billiet (eds.) Cross-Cultural Analysis: Methods and Applications, New York: Routledge.

Vandenberg, R.J. and Lance, C.E. (2000) 'A review and synthesis of the measurement invariance literature: Suggestions, practices, and recommendations for organizational research', Organizational Research Methods 3(1): 4-70.

Welkenhuysen-Gybels, J. (2004) 'The performance of some observed and unobserved conditional invariance techniques for the detection of differential item functioning', Quality \& Quantity 38(6): 681-702.

Welkenhuysen-Gybels, J. and Billiet, J. (2002) 'A comparison of techniques for detecting cross-cultural inequivalence at the item level', Quality \& Quantity 36(3): 197-218.

\section{About the Authors}

Gal Ariely is a visiting scholar at the Nanovic Institute for European Studies, University of Notre Dame. He received his Ph.D. in Political Science at the University of Haifa in 2009. His research interests focus on comparative citizenship and national identity.

Eldad Davidov is a Professor of Sociology at the University of Zurich, Switzerland. His research interests are applications of structural equation modelling to survey data, especially in crosscultural and longitudinal research. He has published several papers on the topic recently in Political Analysis, Social Science Research, International Journal of Public Opinion Research and Public Opinion Quarterly. 